\title{
Parametric autoresonant excitation of the nonlinear Schrödinger equation
}

\author{
L. Friedland* \\ Racah Institute of Physics, Hebrew University of Jerusalem, Jerusalem 91904, Israel \\ A. G. Shagalov ${ }^{\dagger}$ \\ Institute of Metal Physics, Ekaterinburg 620990, Russian Federation \\ and Ural Federal University, Mira 19, Ekaterinburg 620002, Russian Federation
}

(Received 21 April 2016; published 18 October 2016)

\begin{abstract}
Parametric excitation of autoresonant solutions of the nonlinear Schrodinger (NLS) equation by a chirped frequency traveling wave is discussed. Fully nonlinear theory of the process is developed based on Whitham's averaged variational principle and its predictions verified in numerical simulations. The weakly nonlinear limit of the theory is used to find the threshold on the amplitude of the driving wave for entering the autoresonant regime. It is shown that above the threshold, a flat (spatially independent) NLS solution can be fully converted into a traveling wave. A simplified, few spatial harmonics expansion approach is also developed for studying this nonlinear mode conversion process, allowing interpretation as autoresonant interaction within triads of spatial harmonics.
\end{abstract}

DOI: 10.1103/PhysRevE.94.042216

\section{INTRODUCTION}

Controlled excitation of waves is an important problem of basic and applied research. Driving a system by an external resonant wave in many cases yields the desired excitation. However, typically, as the driven wave amplitude becomes large, the excitation saturates because the nonlinearity of the system shifts the wave frequency and destroys the resonance condition. Autoresonance is one approach to overcome this limitation using a salient property of many nonlinear waves to stay in resonance with the driving perturbation as parameters are slowly varying in time and/or space. For example, by slowly varying the driving frequency in the autoresonant regime, one can reach much higher excitations, as the driven wave automatically adjusts its amplitude to stay in a continuing resonance with the drive. The idea of autoresonance originated in particle accelerators [1,2], and now there exists a large number of applications of autoresonance in many fields of physics (see Ref. [3] and references therein). Most of the applications to autoresonant nonlinear waves involve a direct resonance approach, were the driven system starts in some equilibrium and the external driving wave passes a resonance with the desired excitation. The frequency (and the phase) of the driven wave in this case follows that of the driving wave continuously. In this work, we focus on a different class of problems, where instead of a direct drive, one oscillates a parameter in the nonlinear wave system, i.e., we study excitation involving the parametric resonance. This phenomenon (see pioneering work by Michael Faraday [4]) plays an important role in many problems of nonlinear wave dynamics and, in contrast to the direct resonance, allows frequency conversion, i.e., excitation of waves having frequency different from that of the drive. Examples include nonlinear optics [5,6], condensed matter [7-9], fluid mechanics [10,11], and plasmas [12]. In contrast to the direct drive approach, the wave parametric autoresonance

\footnotetext{
*lazar@mail.huji.ac.il

${ }^{\dagger}$ shagalov@imp.uran.ru
}

was studied in two applications only: the excitation and control of the diocotron mode in a nonneutral plasma [13] and formation of the Faraday waves [10,11]. In this paper we extend the parametric autoresonance idea to a driven nonlinear Schrodinger (NLS) equation

$$
i \psi_{t}+\psi_{x x}+\left(2 \sigma|\psi|^{2}-\mu \cos \varphi\right) \psi=0,
$$

where $\sigma=1$ or -1 , the driving amplitude $\mu \ll 1, \varphi=k x-$ $\int \omega d t, k=$ const, and the driving frequency $\omega(t)=-\varphi_{t}$ is a slow function of time. In the case of a fixed driving frequency, the parametric resonance in Eq. (1) has been studied in Refs. $[6,7,9,14,15]$. The slow frequency chirp in Eq. (1) introduces a new important ingredient in the problem allowing stable excitation and control of NLS solutions, which is the main subject of this study.

The NLS equation is one of the most important wave equations of nonlinear physics. It describes a large variety of phenomena ranging from light propagation in nonlinear fibers [5], nonlinear Langmuir waves in plasmas [16], gravity waves on the surface of deep water [17], Bose-Einstein condensates (BEC) $[18,19]$, and more. The unperturbed NLS equation has different solutions ranging from plane waves to solitons and more complicated multiphase solutions [20]. The problem of excitation of a particular type of solution is therefore important in applications. Starting in a simple experimentally realizable initial state is important in successful formation of different NLS waves. The passage through resonance with these initial states and transition to autoresonance in NLS systems using a direct drive was used previously for controlled excitation of a variety of NLS solutions [21-23]. In the present work we address a similar problem in the parametrically driven NLS case. In many wave systems, the NLS equation describes modulations of the amplitude of the wave in a nonlinear medium. Therefore, the most natural and easily realizable initial state is a constant amplitude wave (corresponding to a flat, $x$-independent NLS equilibrium), and, therefore, in this work, we will focus on a flat initial state. The parametric driving in the case of a wave in a nonlinear medium can be 
induced by modulating the linear dispersion of the background. In contrast, in BEC systems, the parametric driving would involve modulation of the trapping potential.

The scope of the paper will be as follows. In Sec. II we will present a weakly nonlinear theory of passage through resonance in our parametrically driven system. We will show that if starting in a flat equilibrium, as the driving frequency passes the resonance with this equilibrium, one enters the autoresonant stage of Eq. (1), as the solution phase-locks to the drive, provided the driving amplitude $\mu$ exceeds a threshold, which scales with the driving frequency chirp rate $\alpha=d \omega / d t$ as $\alpha^{3 / 4}$. We will compare this prediction with simulations and show that above the threshold, as the variation of the driving frequency continues, the nonlinear resonance in the system is preserved, while the initial wave is transformed into a different nonlinear wave. This transformation in invertible by simply changing the direction of variation of the driving frequency, i.e., the nonlinear waves involved in the interaction are fully controlled by the frequency of the driving perturbation. The understanding of the nonlinear mode conversion of waves in the problem comprises the main goal of this work. Section III will be devoted to fully nonlinear theory of this autoresonant mode conversion. The phenomenon belongs to the class of problems characterized by two very different time scales, the fast scale associated with the period of the NLS wave and the slow scale due to the driving frequency chirp. The classical Bogolubov-Krylov-Mitropolski averaging method [24] was developed for two scale problems in the case of finitedimensional systems of classical mechanics and oscillation theory. Later, an analog of the averaging method for partial differential equations having Lagrangian form was developed by Whitham [25]. The NLS equation belongs to the latter class of problems and, thus, the Whitham's averaged Lagrangian approach will be used in the present work. We will also verify the predictions of this theory by simulations in Sec. III. In Sec. IV, we suggest a simplified theory of wave interactions in the system using a truncated harmonics expansion. It will be shown that the observed mode transformations can be viewed as nonlinear interactions within triads of harmonics of the driven NLS solution. Finally, our conclusions will be outlined in Sec. V.

\section{WEAKLY NONLINEAR EVOLUTION AND THE THRESHOLD PHENOMENON}

We proceed with studying the solution of the parametrically driven NLS equation (1) subject to a flat ( $x$-independent) initial condition $\psi\left(x, t_{0}\right)=U_{0}>0$. We write this solution in terms of the real amplitude and complex phase, $\psi=U e^{i \theta}$, yielding a system of real equations

$$
\begin{gathered}
U_{t}+\theta_{x x} U+2 \theta_{x} U_{x}=0 \\
\theta_{t} U-U_{x x}+\theta_{x}^{2} U-2 \sigma U^{3}=-\mu U \cos \varphi .
\end{gathered}
$$

This system can be also obtained from the variational principle via the Lagrangian density

$$
L=\frac{1}{2}\left[U_{x}^{2}+U^{2}\left(\theta_{x}^{2}+\theta_{t}\right)\right]-\frac{\sigma}{2} U^{4}+\frac{\mu U^{2}}{2} \cos \varphi .
$$

Next, we assume $U=U(\Theta)$ and $\theta=\xi+V(\Theta)$, where $\Theta=$ $k x-\int \Omega d t$ and $\xi=-\int \Omega_{0} d t$ are fast phases, while both $\Omega$ and $\Omega_{0}$ are slow functions of time and seek phase-locked solutions $\Theta \approx \varphi$ in the driven problem. For constant $\Omega$ and $\Omega_{0}$, this is the so-called one-band NLS solution [20]. Because of the assumed adiabaticity, we apply the Whitham's averaged variational approach [25], i.e., average the Lagrangian density with respect to the fast phase $\Theta$ :

$$
\Lambda=\langle L\rangle_{\Theta}=\Lambda_{0}+\Lambda_{1},
$$

where $\Lambda_{0}$ describes the undriven problem and includes only slow variables, while the perturbed part $\Lambda_{1}=$ $\left\langle\left(\mu U^{2} / 2\right) \cos \varphi\right\rangle_{\Theta}$. We will discuss our general averaging procedure in the next section and here focus on the weakly nonlinear limit only. The weakly nonlinear averaged Lagrangian density is obtained via the harmonic decomposition

$$
\begin{gathered}
U=u_{0}+u_{1} \cos \Theta+u_{2} \cos (2 \Theta), \\
V=-v_{1} \sin \Theta-v_{2} \sin (2 \Theta),
\end{gathered}
$$

where $u_{0}$ is of $O(1), u_{1} \ll 1, v_{1}$ is of $O\left(u_{1}\right)$, and $u_{2}, v_{2}$ are of $O\left(u_{1}^{2}\right)$. The amplitudes of various harmonics are assumed to be slow. A similar weakly nonlinear theory was developed in Ref. [23], studying the direct driving problem. The averaged unperturbed weakly nonlinear Lagrangian density $\Lambda_{0}\left(u_{0}, u_{1}, u_{2}, v_{1}, v_{2} ; \Omega, \Omega_{0}\right)$ from this work is given in the Appendix, and we use this Lagrangian density in the following. To lowest significant order, the perturbing part of the Lagrangian density is

$$
\Lambda_{1} \approx \mu u_{0} u_{1}\langle\cos \Theta \cos \varphi\rangle_{\Theta} \approx \frac{\mu}{2} u_{0} u_{1} \cos \Phi
$$

where we have left only the slow (resonant) contribution, viewing the phase mismatch $\Phi=\Theta-\varphi$ as a slow function of time. Given the averaged Lagrangian density, we take variations with respect to all slow amplitudes, $\Theta$ and $\xi$ yielding, after elimination of the zero and second harmonics, the following system of two slow evolution equations (see the Appendix)

$$
\begin{gathered}
u_{1 t}=-\frac{\varepsilon}{2} \sin \Phi, \\
\Phi_{t}=\alpha t-\sigma \beta u_{1}^{2}-\frac{\varepsilon}{2 u_{1}} \cos \Phi .
\end{gathered}
$$

Here $\quad \varepsilon=\frac{\mu k^{2} U_{0}}{\Omega_{r}^{ \pm}}, \quad \beta=1 / \rho+3 \rho, \quad \rho=\Omega_{r}^{ \pm} / k^{2}, \quad \Omega_{r}^{ \pm}=$ $\pm k \sqrt{k^{2}-4 \sigma U_{0}^{2}}$ are the two linear resonance frequencies in the problem, and we have assumed that the driving frequency $\omega(t)=\Omega_{r}^{ \pm}+\alpha t, \alpha$ being a constant (and small) chirp rate. Nevertheless, it should be noted that the autoresonant evolution of nonlinear systems is very robust, since the phase-locking is not affected by the form of time variation of the driving frequency, as long as this variation is sufficiently slow, while the sign of the frequency chirp rate for capture into resonance is determined by the signs of the nonlinearity and frequency of the oscillatory system. As in the direct driving case in Ref. [23], Eqs. (9) and (10) describe the standard weakly nonlinear capture into autoresonance problem and predict efficient phase locking (a near stationarity of $\Phi$ ) prior to the driver passing the linear resonance. After the passage, 

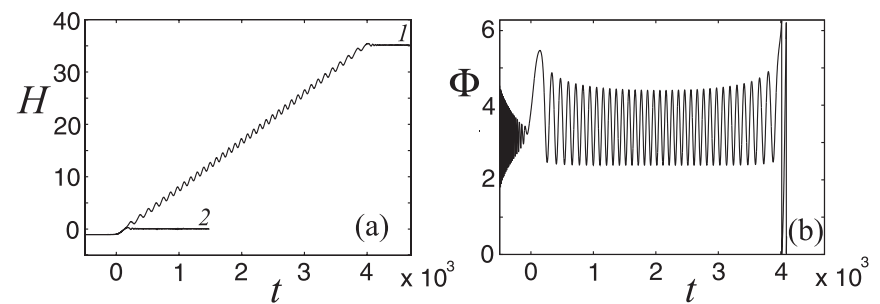

FIG. 1. (a) The autoresonant growth of the energy $H$ in time (line 1) for $\mu=0.00225$ just above the threshold and the small energy jump after passage through the linear resonance (line 2) for $\mu=0.00223$. The numerical threshold value is $\mu_{t h}=0.00224$. (b) The evolution of the phase mismatch $\Phi$ for $\mu=0.00225$; in all cases $\sigma=1, \alpha=10^{-3}$, $k=6$, and $U_{0}=1$.

the phase locking continues provided the driving amplitude exceeds the threshold $\left|\varepsilon_{t h}\right|=0.82|\alpha|^{3 / 4}|\beta|^{-1 / 2}$ [3] or

$$
\left|\mu_{t h}\right|=\frac{0.82|\alpha|^{3 / 4}}{U_{0}\left|1 / \rho^{3}+3 / \rho\right|^{1 / 2}} .
$$

It is important at this stage to discuss the differences of the autoresonant trapping problems in four cases corresponding to passage trough two resonant frequencies $\Omega_{r}^{ \pm}$for different signs of $\sigma$. These cases differ by initial trapping stages, where we solve Eqs. (9) and (10) subject to $u_{1} \rightarrow 0$ as $t \rightarrow-\infty$ and, therefore, at large negative times $\sin \Phi \approx 0$ and $u_{1} \approx$ $-\frac{\varepsilon}{2 \alpha|t|} \cos \Phi$. Then we phase lock at either $\Phi \approx \pi$ or $\Phi \approx 0$ $[\bmod (2 \pi)]$ if the sign of the ratio $\varepsilon / \alpha$ (the sign of $\alpha \Omega_{r}^{ \pm}$) is positive or negative, respectively. On the other hand, above the threshold, after passage through resonance (large positive times), the phase locking continues and the amplitude grows as $u_{1}^{2} \approx \sigma \alpha t / \beta$. This requires $\sigma \alpha / \beta$ to be positive. Thus, for $\sigma=$ 1 , one must use positive or negative chirp rate when passing through $\Omega_{r}^{+}$or $\Omega_{r}^{-}$, respectively, and vice versa for $\sigma=-1$. Furthermore, for $\sigma=1$, the locking of $\Phi$ is always around $\pi$ $[\bmod (2 \pi)]$, while for $\sigma=-1, \Phi$ locks around zero. Note that in all these cases, the threshold (11) is the same.

For testing these predictions we solved NLS equation (1) numerically in the range $[0, D]$ with periodic boundary conditions, while $D=2 \pi / k$ was defined by the wave number of the driving perturbation. We used a standard spectral method (see, e.g., Ref. [26]) in simulations via Fourier transform representation

$$
\psi(x, t) \approx \sum_{n=-N / 2}^{N / 2-1} \psi_{n}(t) e^{i k_{n} x}, \quad k_{n}=\frac{2 \pi}{D} n,
$$

where the number of modes $N$ in computations was varied up to 256 . Figure 1 shows the energy

$$
H(t)=\int_{0}^{D}\left(|\nabla \psi|^{2}-\sigma|\psi|^{4}\right) d x
$$

versus time as found in simulations in $\sigma=1$ case. We start at $t=-T_{0}$ in the figure with the flat initial state $(n=0)$ of amplitude $\left|\psi\left(x,-T_{0}\right)\right|=U_{0}=1$, decrease $(\alpha<0)$ the driving frequency linearly in time, and cross the resonance frequency $\Omega_{r}^{-}$at $t=0$. If the amplitude $\mu$ of the driving perturbation is less than some threshold value, we observe a typical linear resonance response, as the energy experiences a
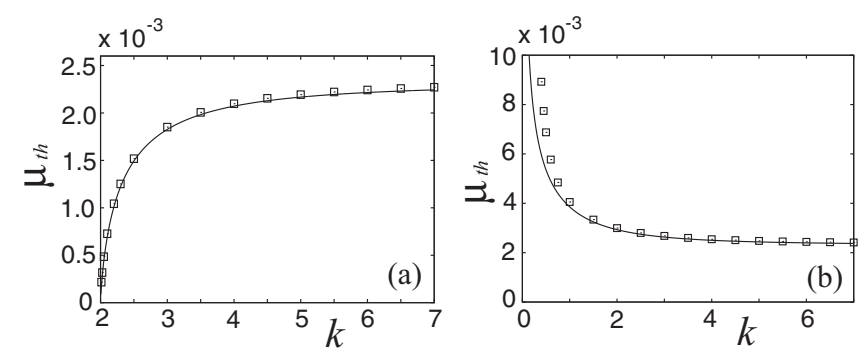

FIG. 2. The theoretical (solid lines) and numerical (squares) thresholds $\mu_{t h}$ vs $k$ for $\sigma=1$ (a) and $\sigma=-1$ (b), $|\alpha|=10^{-3}$, and $U_{0}=1$.

small jump after crossing the linear resonance (line 2 in the left panel in the figure). But, if the driving amplitude exceeds the threshold, the energy evolution changes drastically (line 1 in the left panel) even if $\mu$ is just above the threshold. The energy increases significantly and saturates at a much higher level in the final stage. At the same time, above the threshold, we observe a continuing phase locking of the phase mismatch $\Phi$ around $\pi$ (see the right panel in Fig. 1) during and after crossing the linear resonance, as predicted earlier. The saturation of the energy was observed only after the phase locking was destroyed. Such behavior is characteristic of autoresonance and is consistent with the theory described above. The comparison of the theoretical threshold (11) for $\sigma= \pm 1,|\alpha|=10^{-3}, U_{0}=1$, and different values of $k$ with the numerically found thresholds are presented in Fig. 2, illustrating a very good agreement.

Additional details on the autoresonance in our system are provided by examining the time evolution of different modes in series (12) as illustrated in Fig. 3 for the example in the left and right panels in Fig. 1 above the threshold. At the time of the linear resonance $(t=0)$, we observe a large amplitude $n=0$ mode, corresponding to the initial flat wave of unit amplitude. The new, small amplitude $n=-1$ mode is added to the $n=0$ mode in the initial phase locking stage, the combination comprising the initial autoresonant nonlinear NLS wave. At the intermediate times, the $n=-1$ mode grows at the cost of the flat mode because the NLS equation (1) conserves $I=$ $\sum\left|\psi_{n}\right|^{2}$. In the final stage, most of the energy is transformed to the resonant $n=-1$ mode. Shortly afterward, the phase locking is destroyed and the mode conversion process comes to an end. Figure 3 also demonstrates that throughout the process only a small number of modes is involved in this autoresonant mode conversion.
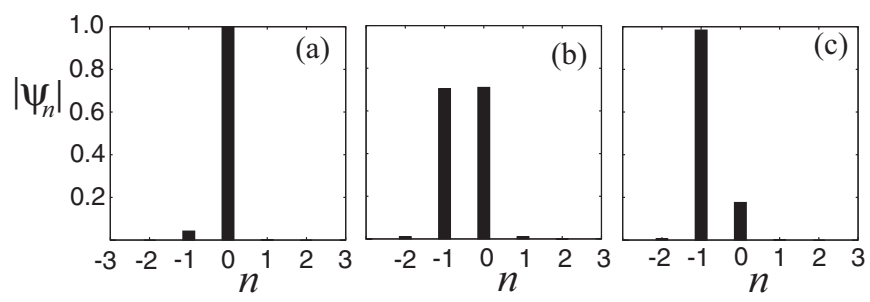

FIG. 3. Autoresonant mode conversion at different times for the example above the threshold ( $\mu=0.00225)$ in Fig. 1. (a) $t=0$, (b) $t=2000$, and (c) $t=4000$. 


\section{FULLY NONLINEAR AVERAGED VARIATIONAL THEORY}

In this section we discuss a fully nonlinear autoresonant evolution in our problem focusing on passage through the linear resonance by down chirping the driving frequency $\omega(t)=\Omega_{r}+\alpha t, \alpha<0$ and starting with flat initial conditions $\psi(x, 0)=U_{0}$. As discussed above, this case corresponds to the resonant frequency

$$
\Omega_{r}=-\sigma k \sqrt{k^{2}-4 \sigma U_{0}^{2}}
$$

with phase locking on either $\Phi \approx 0$ or $\pi$ for $\sigma=1$ or -1 , respectively.

Our fully nonlinear theory is again based on the Whitham's averaged variational principle. This approach was developed for the direct driving case in Ref. [27], and we use similar developments in the parametric drive case. We proceed from Lagrangian density (4), express $\theta_{x}=k V_{\theta}$ and $\theta_{t}=-\Omega_{0}-$ $\Omega V_{\theta}=-\Omega_{0}-(\Omega / k) V_{x}$, and rewrite the unperturbed part $L_{0}$ of the Lagrangian density in the form

$$
L_{0}=\frac{1}{2}\left[U_{x}^{2}+U^{2} V_{x}^{2}-\frac{\Omega}{k} U^{2} V_{x}-\Omega_{0} U^{2}-\sigma U^{4}\right],
$$

while, as before, the perturbed part is

$$
L_{1}=\frac{\mu U^{2}}{2} \cos \varphi
$$

We observe that $V$ in the unperturbed problem is a cyclic variable, and, thus, the canonical momentum $P_{V}=\partial L_{0} / \partial V_{x}=$ $U^{2}\left(V_{x}-\Omega / 2 k\right) \equiv B$ is conserved. In the autoresonantly perturbed problem, $B$ becomes a slow function of time. We define the second canonical momentum $P_{U}=\partial L_{0} / \partial U_{x}=U_{x}$ and write the Hamiltonian $H_{0}=P_{U} U_{x}+P_{V} V_{x}-L_{0}$, which after some algebra yields the second slow variable in the perturbed problem

$$
H_{0}=\frac{1}{2}\left(P_{U}^{2}+\frac{B^{2}}{U^{2}}\right)+\frac{R}{2} U^{2}+\frac{\sigma}{2} U^{4}+\frac{\Omega}{2 k} B,
$$

where

$$
R=\Omega_{0}+\frac{1}{4}\left(\frac{\Omega}{k}\right)^{2}
$$

Then, by introducing a more convenient slow variable $A=$ $H_{0}-\frac{\Omega}{2 k} B$, we have

$$
A=\frac{1}{2} P_{U}^{2}+V_{\mathrm{eff}}(U)
$$

where

$$
V_{\text {eff }}=\frac{1}{2} \frac{B^{2}}{U^{2}}+\frac{R}{2} U^{2}+\frac{\sigma}{2} U^{4}
$$

This result allows us to interpret the unperturbed evolution of $U$ as an oscillation of a quasiparticle in the effective potential, while $A$ plays a role of the energy. In the driven problem, we proceed from the equilibrium $U=U_{0}$ (at the minimum of the quasipotential) and excite the autoresonant evolution of the quasiparticle, as the driving frequency passes the resonance, while both $A$ and $B$ slowly vary in time. Initially $V_{\text {eff }}^{\prime}\left(U_{0}\right)=0$ yielding initial $B^{2}=U_{0}^{4}\left(R+2 \sigma U_{0}^{2}\right)$, while

$$
V_{\mathrm{eff}}^{\prime \prime}\left(U_{0}\right)=4 R+12 \sigma U_{0}^{2}=k^{2} \text {. }
$$

Then the resonant frequency is indeed given by Eq. (14) (recall that the choice of the sign of the frequency is given by $\sigma$, and here we discuss the autoresonance with a down chirped driving frequency).

For studying autoresonant evolution of $A, B, \Omega$, and $\Omega_{0}$, we seek a slow averaged Lagrangian density. To this end, we first rewrite

$$
L_{0}=P_{U} U_{x}+P_{V} V_{x}-H_{0}=-A-\frac{\Omega}{2 k} B+P_{U}^{2}+B V_{x} .
$$

Next, according to the Whitham's procedure [25], we fix the slow time and average $L_{0}$ over $\Theta$ to get

$$
\Lambda_{0}=-A-\frac{\Omega}{2 k} B+k J(A, B, R),
$$

where

$$
J=\frac{1}{2 \pi} \int_{0}^{2 \pi} P_{U}^{2} d \Theta=\frac{1}{2 \pi} \oint \sqrt{A-V_{\mathrm{eff}}(B, R, U)} d U
$$

For averaging the perturbed Lagrangian density, we first expand $U(\Theta)=u_{0}+u_{1} \cos \Theta+u_{2} \cos (2 \Theta)+\cdots$ and substitute it into (16). Then, upon averaging,

$$
\Lambda_{1}=\frac{1}{2} \mu u_{0} u_{1} \cos \Phi .
$$

Finally, we use the full averaged Lagrangian density $\Lambda=$ $\Lambda_{0}+\Lambda_{1}$ to write the slow evolution equations in the problem. The variations with respect to $A$ and $B$ yield

$$
k J_{A}-1=-\frac{1}{2} \mu\left(a_{0} a_{1}\right)_{A} \cos \Phi
$$

and

$$
k J_{B}-\frac{\Omega}{2 k}=-\frac{1}{2} \mu\left(a_{0} a_{1}\right)_{B} \cos \Phi .
$$

The variations with respect to $\xi=-\int \Omega_{0} d t$ and $\Theta$ yield additional two equations

$$
\frac{d}{d t}\left(k J_{\Omega_{0}}\right)=0
$$

and

$$
\frac{d}{d t}\left(k J_{\Omega}-B / 2 k\right)=\frac{1}{2} \mu\left(a_{0} a_{1}\right) \sin \Phi .
$$

Next, we focus on the approximate, slow autoresonant quasi-equilibrium. To lowest significant order in the driving amplitude $\mu$, this equilibrium is described by the set

$$
\begin{gathered}
k J_{A}-1 \approx 0, \\
k J_{B}-\frac{\Omega}{2 k} \approx 0, \\
k J_{\Omega_{0}}=k J_{R}=C, \quad C=\text { const },
\end{gathered}
$$

which by adding the persistent resonance condition

$$
\Omega \approx \omega
$$

yields the slow autoresonant evolution of $A, B, \Omega$, and $\Omega_{0}$. The convenient numerical procedure for solving this algebraic 


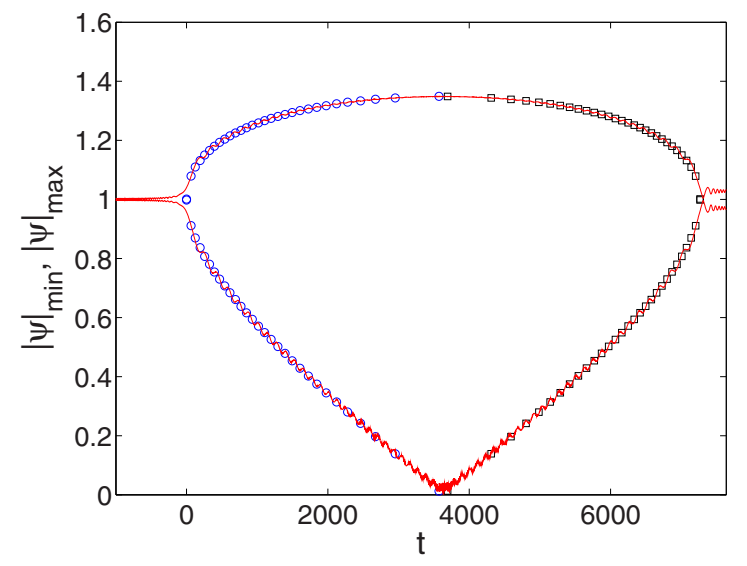

FIG. 4. Autoresonant dynamics in $\sigma=-1$ case. $|\psi|_{\text {min }}$ and $|\psi|_{\max }$ vs time. Solid red lines show numerical simulations, circles and squares represent results from the quasi-equilibrium theory. The parameters are $k=3, \alpha=-5 \times 10^{-4}$, and $U_{0}=1$.

system is as follows. The initial condition yields $C=-U_{0}^{2} / 2$, while initially

$$
B_{0}=\frac{\sigma}{2} \sqrt{k^{2}-4 \sigma U_{0}^{2}}
$$

and from (21)

$$
R_{0}=k^{2} / 4-3 \sigma U_{0}^{2} .
$$

By making small steps in $R$, starting from $R_{0}$, one solves Eqs. (A6) and (A8) for $A$ and $B$ by iterations. When these variables are know, one finds $\Omega$ from (31a) and

$$
\Omega_{0}=R-\frac{1}{4}\left(\frac{\Omega}{k}\right)^{2}
$$

from the definition of $R$. Then one also knows the driving frequency $\omega(t) \approx \Omega$ from Eq. (33), i.e., associates all these variables and parameters with a particular time of evolution. From the time evolution of the slow variables we also know the instantaneous effective potential in the problem and can find the oscillations of $U$ and $V$ at a given time. We illustrate a calculation of this type in Figs. 4 and 5, showing (by circles) $\max |U|$ and $\min |U|$ as functions of the driving frequency for $\sigma=-1$ and +1 , respectively. One can see that the results agree very well with those of full numerical simulations (red lines) of the driven system. We calculate the quasi-equilibrium (circles) in the figures until min $|U|$ approaches zero. At this time, $B$ passes through zero and should become negative, but $k J_{B}$ remains finite, and a different approach must be taken to cross this transition point. Such an approach is described next.

The idea is based on the results of the full simulations showing that with the decrease of the driving frequency, the initial flat solution $\psi=U_{0} e^{i 2 \sigma U_{0}^{2}\left(t-t_{0}\right)}$ at some time $t_{f}$ almost fully converts into a traveling wave $\psi \approx a e^{-i \sigma \varphi}$, with $|a| \approx U_{0}$. Beyond $t_{f}$, the autoresonance discontinues. Because of the time reversibility of our dynamical problem, this mode conversion phenomenon indicates that the process can be reversed, i.e., by starting from the traveling wave at $t_{f}$, one can autoresonantly convert it into a flat solution by going backward in time, i.e., by increasing the driving frequency.

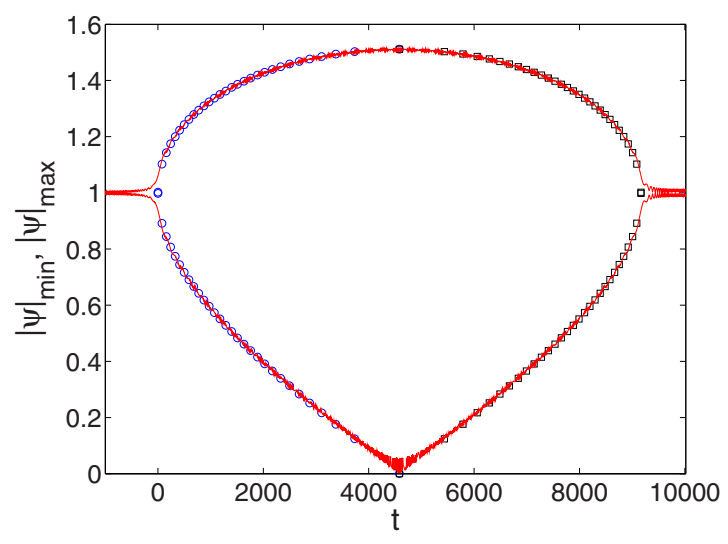

FIG. 5. Autoresonant dynamics in $\sigma=+1$ case. $|\psi|_{\text {min }}$ and $|\psi|_{\max }$ versus time. Solid red lines show numerical simulations, circles and squares represent results from the quasi-equilibrium theory. Other parameters are as in Fig. 4.

Let us consider this process in more detail. The first step in this direction is to find the resonant driving frequency for the initial traveling wave solution. The method is the same as above for calculating the resonant frequency for the flat solution, i.e., the Lagrangian approach. We again seek solutions of form $\psi=U(\Theta) e^{i \theta}$, with $\Theta=k x-\int \Omega^{\prime} d t$, but $\theta=-\sigma k x-\int \Omega_{0}^{\prime} d t+V(\Theta)$. Then similar developments as above yield the same unperturbed dynamical system [compare to (19) and (20)]

$$
A^{\prime}=\frac{1}{2} P_{U}^{2}+V_{\text {eff }}(U)
$$

with the same form of the effective potential

$$
V_{\mathrm{eff}}=\frac{1}{2} \frac{B^{\prime 2}}{U^{2}}+\frac{R^{\prime}}{2} U^{2}+\frac{\sigma}{2} U^{4}
$$

but now $A^{\prime}=H_{0}^{\prime}-\sigma k B^{\prime}-B^{\prime} \Omega^{\prime} / 2 k, \quad B^{\prime}=U^{2}(-\sigma k-$ $\left.\Omega^{\prime} / 2 k+V_{x}\right)$,

$$
R^{\prime}=\sigma \Omega^{\prime}+\Omega_{0}^{\prime}+\frac{\Omega^{\prime 2}}{4 k^{2}}
$$

and initially $\Omega_{0}^{\prime}=k^{2}-2 \sigma U_{0}^{\prime 2}$. As before, the initial $U_{0}$ corresponds to the minimum of $V_{\text {eff }}$, yielding [via similar developments as for (14)] the new resonant frequency

$$
\Omega_{r}^{\prime}=-\sigma\left(2 k^{2}-k \sqrt{k^{2}-4 \sigma U_{0}^{2}}\right) .
$$

By comparing this result with the simulations one can see that the complete mode conversion in the previous setting is indeed achieved when the driving frequency reaches $\Omega_{r}^{\prime}$, i.e., at

$$
t_{f}=\frac{\Omega_{r}-\Omega_{r}^{\prime}}{\alpha}=\frac{2 \sigma}{\alpha}\left(k^{2}-k \sqrt{k^{2}-4 \sigma U_{0}^{2}}\right) .
$$

Now we use the traveling wave initial condition and increase the driving frequency by going backward in time, i.e., use

$$
\omega^{\prime}(t)=\omega_{r}^{\prime}+\alpha\left(t_{f}-t\right)
$$

with the goal to mode convert into a flat solution. Note that using (39), one finds that $\omega_{d}^{\prime}(t)=\omega_{d}(t)$ for all times. The details of the backward excitation process can be again dealt within the Whitham's averaged Lagrangian approach presented below. 
By using the same developments as in the previous case, we get the averaged Lagrangian density of the same form

$$
\Lambda^{\prime}=-A^{\prime}-\frac{\Omega^{\prime}}{2 k} B^{\prime}+k J\left(A^{\prime}, B^{\prime}, R^{\prime}\right)+\frac{1}{2} \mu a_{0} a_{1} \cos \Phi,
$$

but with $R^{\prime}, B^{\prime}$, and $A^{\prime}$ defined differently. This Lagrangian density yields a system of equations describing the slow autoresonant equilibrium:

$$
\begin{gathered}
k J_{A^{\prime}}-1 \approx 0, \\
k J_{B^{\prime}}-\frac{\Omega^{\prime}}{2 k} \approx 0, \\
k J_{\Omega_{0}^{\prime}}=k J_{R^{\prime}}=C^{\prime}, \quad C^{\prime}=\text { const },
\end{gathered}
$$

subject to the persistent locking condition

$$
\Omega^{\prime} \approx \omega^{\prime} \text {. }
$$

Here $C^{\prime}=-U_{0}^{2} / 2$ is given by the initial conditions. Furthermore, one finds that initially, $R_{0}^{\prime}=R_{0}$, while $B_{0}^{\prime}=-B_{0}$ and, therefore, $A_{0}^{\prime}=A_{0}$. As before, the numerical procedure for solving this problem is to find $A^{\prime}$, and $B^{\prime}$ from (42) and (44) for a set of values of $R^{\prime}$ starting from $R_{0}^{\prime}$. But this is the same system studied previously, and, therefore, it yields the same $A^{\prime}=A, R^{\prime}=R$, while $B^{\prime}=-B$ for the same $R^{\prime}=R$. Consequently, the quasipotential $V_{\text {eff }}$ will be the same (it depends on $B^{\prime 2}$ ) and yields the same spatial oscillation of $U$, i.e., the same $\max (|\psi|)$ and $\min (|\psi|)$ and $\Omega^{\prime}=\Omega$ [from (43)]. The only difference will be in the dependence of $\max (|\psi|)$ and $\min (|\psi|)$ on time, because of a different time dependence of $\Omega^{\prime}$ via Eq. (45). As the driving frequency in this case increases, $\Omega^{\prime}$ will increase, while, as before, $|B|$ will decrease and approach zero at some time $t_{t r}$, signaling the transition to the previous case. This analysis explains the symmetry of the dependence of $\max (|\psi|)$ and $\min (|\psi|)$ on time around $t_{t r}$ (the squares in Figs. 4 and 5 represent the inverse transition process), as also observed in the simulations. Because of this symmetry $t_{t r}=t_{f} / 2$ [see Eq. (39)], in agreement with simulations. This analysis also explains the complete autoresonant mode conversion from the flat to the traveling wave solution and vice versa.

\section{TRUNCATED HARMONICS EXPANSION}

Despite the generality of the full nonlinear Lagrangian approach described above, its practical implementation requires numerical solution of a system of complex algebraic equations by iterations allowing to find the autoresonant quasi-equilibrium solution. Here we present a less accurate, but simpler approach, yielding the autoresonant solution and allowing us to study its stability. The approach is based on the results of full simulations above, showing that in the process of mode conversion from the flat to the traveling wave solution, only a small number of harmonics of the solution are involved throughout the transformation process. This suggests an approach to the problem based on few harmonic decomposition, which is described next. We seek the following truncated harmonic expansion of the solution

$$
\psi \approx a_{0}+a_{+} e^{i \varphi}+a_{-} e^{-i \varphi}+b_{+} e^{2 i \varphi}+b_{-} e^{-2 i \varphi} .
$$

Here we do not assume the smallness of the amplitudes $a_{ \pm}$ and $b_{ \pm}$throughout the evolution process. We substitute this solution into Eq. (1) and, separating contributions of different harmonics, obtain the following set of equations:

$$
\begin{gathered}
i a_{0 t}+2 \sigma N_{0}=\frac{\mu}{2}\left(a_{-}+a_{+}\right), \\
i a_{-t}+\left(-\omega-k^{2}\right) a_{-}+2 \sigma N_{-}=\frac{\mu}{2}\left(a_{0}+b_{-}\right), \\
i a_{+t}+\left(\omega-k^{2}\right) a_{+}+2 \sigma N_{+}=\frac{\mu}{2}\left(a_{0}+b_{+}\right), \\
i b_{-t}-2\left(\omega+2 k^{2}\right) b_{-}+2 \sigma M_{-}=\frac{\mu}{2} a_{-}, \\
i b_{+t}+2\left(\omega-2 k^{2}\right) b_{+}+2 \sigma M_{+}=\frac{\mu}{2} a_{+},
\end{gathered}
$$

where, using the conservation law, $\left|a_{0}\right|^{2}+\left|a_{-}\right|^{2}+\left|a_{+}\right|^{2}+$ $\left|b_{-}\right|^{2}+\left|b_{+}\right|^{2}=U_{0}^{2}$, and

$$
\begin{aligned}
N_{0}= & 2\left(a_{-}^{*} a_{+} b_{-}+a_{-} a_{+}^{*} b_{+}+a_{0}^{*} a_{-} a_{+}+a_{0}^{*} b_{-} b_{+}\right) \\
& +a_{0}\left(2 U_{0}^{2}-\left|a_{0}\right|^{2}\right)+a_{-}^{2} b_{-}^{*}+a_{+}^{2} b_{+}^{*}, \\
N_{-}= & 2\left(a_{0}^{*} a_{+} b_{-}+a_{+}^{*} b_{+} b_{-}+a_{0} a_{+} b_{+}^{*}+a_{0} a_{-}^{*} b_{-}\right) \\
& \times a_{-}\left(2 U_{0}^{2}-\left|a_{-}\right|^{2}\right)+a_{0}^{2} a_{+}^{*}, \\
N_{+}= & 2\left(a_{0}^{*} a_{-} b_{+}+a_{-}^{*} b_{-} b_{+}+a_{0} a_{-} b_{-}^{*}+a_{0} a_{+}^{*} b_{+}\right) \\
& +a_{+}\left(2 U_{0}^{2}-\left|a_{+}\right|^{2}\right)+a_{0}^{2} a_{-}^{*}, \\
M_{-}= & a_{-}\left(2 U_{0}^{2}-\left|b_{-}\right|^{2}\right)+2\left(a_{0} a_{-} a_{+}^{*}+a_{-} a_{+} b_{+}^{*}\right) \\
& +a_{0}^{2} b_{+}^{*}+a_{-}^{2} a_{0}^{*} .
\end{aligned}
$$

Figures 6 and 7 show the absolute values $A_{0}=\left|a_{0}\right|$, $A_{ \pm}=\left|a_{ \pm}\right|, B_{ \pm}=\left|b_{ \pm}\right|$of different harmonics and their complex relative phases $\left(\varphi_{ \pm}, \theta_{ \pm}\right)=\arg \left(a_{ \pm}, b_{ \pm}\right)-\arg \left(a_{0}\right)$ obtained by solving Eqs. (47)-(51) subject to initial conditions
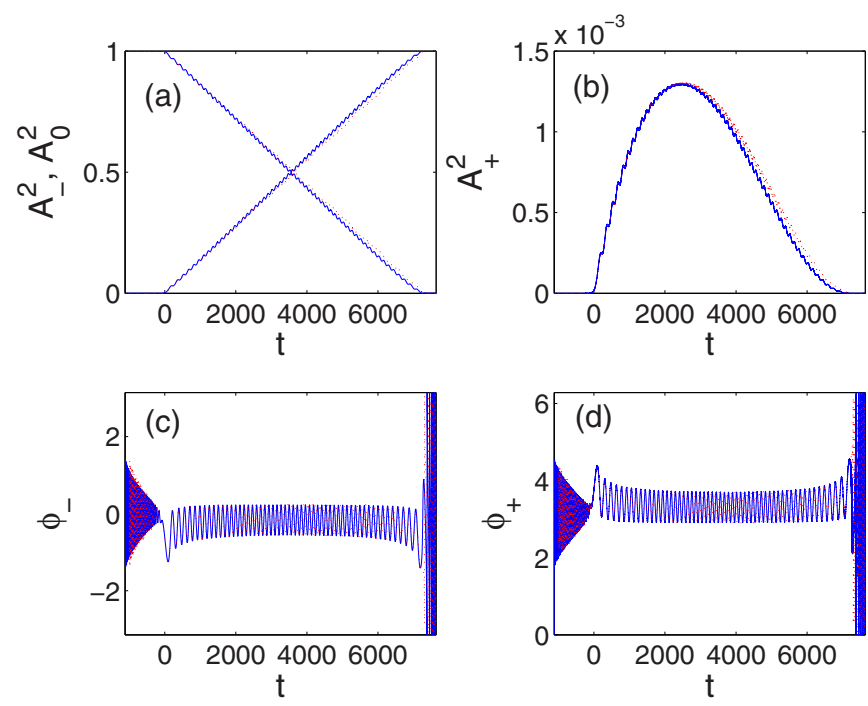

FIG. 6. The amplitudes (a, b) and the relative phases (c, d) of $a_{0}, a_{-}, a_{+}$harmonics in the $\sigma=1$ example $\left(U_{0}=1, k=3\right.$, $\mu=0.002$, and $\alpha=-0.0005)$. The results of full simulations are shown by red dashed lines. 

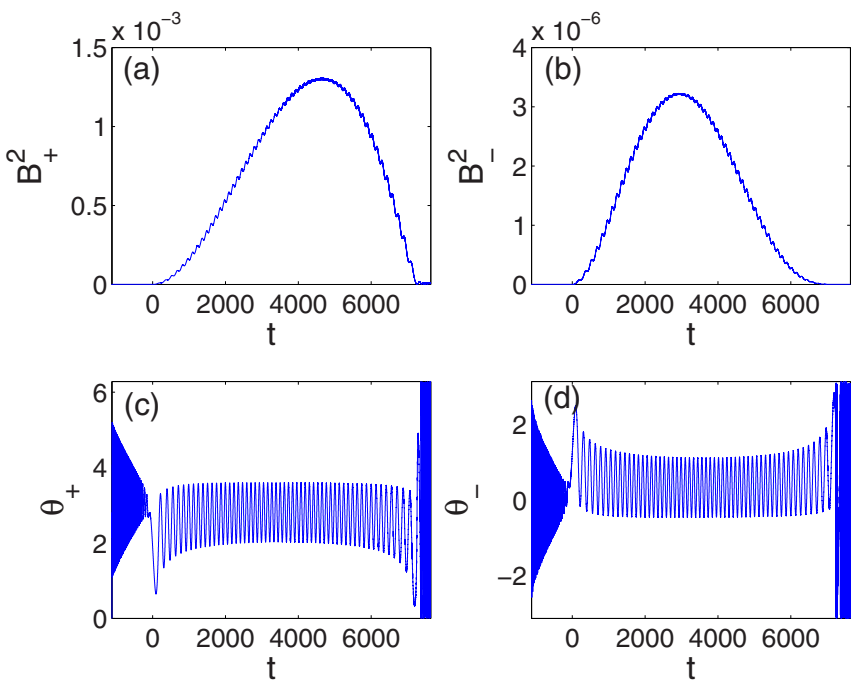

FIG. 7. The amplitudes $(a, b)$ and the relative phases (c, d) of $b_{+}, b_{-}$harmonics in the example in Fig. 6.

$a_{0}=U_{0}=1, a_{ \pm}=b_{ \pm}=0$ for parameters $\sigma=1, k=3$, $\mu=0.002$, and $\omega=\omega_{r}+\alpha t$, where $\alpha=-0.0005, \omega_{r}$ being the linear resonance frequency [see Eq. (14)]. One can see the efficient phase locking (autoresonance) of all harmonics to the drive at either 0 or $\pi[\bmod (2 \pi)]$ after passage through the linear resonance and that the initial flat solution $a_{0}$ is fully transformed to the traveling wave $a_{-} e^{-i \varphi}$ in this example. We also compare the approximate solutions in Fig. 6 with full numerical simulations, illustrating an excellent agreement. Note that all harmonics but $a_{0}$ and $a_{-}$remain small throughout the mode transformation process. Nonetheless, some of these small harmonics cannot be neglected, because they define the resonant frequencies in the problem. Indeed, in the initial stage of mode conversion process, one can neglect the second harmonics $b_{ \pm}$, but must leave $a_{ \pm}$in addition to $a_{0}$. This yields a simpler set for the triad $a_{0}, a_{ \pm}$:

$$
i a_{0 t}+2\left[a_{0}\left(2 U_{0}^{2}-\left|a_{0}\right|^{2}\right)+2 a_{0}^{*} a_{+} a_{-}\right]=\frac{\mu}{2}\left(a_{+}+a_{-}\right),
$$

$$
i a_{+t}+a_{+}\left(\omega-k^{2}+4 U_{0}^{2}\right)-2\left(\left|a_{+}\right|^{2} a_{+}-a_{0}^{2} a_{-}^{*}\right)=\frac{\mu}{2} a_{0},
$$

$i a_{-t}+a_{-}\left(-\omega-k^{2}+4 \sigma U_{0}^{2}\right)-2\left(\left|a_{-}^{2}\right| a_{-}-a_{0}^{2} a_{+}^{*}\right)=\frac{\mu}{2} a_{0}$.

In the undriven case, by linearization around the initial $a_{0}=$ $U_{0}$, Eq. (56) yields $a_{0}=U_{0} e^{i 2 U_{0}^{2}\left(t-t_{0}\right)}$. Then, by seeking constant amplitude solutions $a_{ \pm}=\widetilde{a}_{ \pm} e^{i U_{0}^{2}\left(t-t_{0}\right)}$ in the unperturbed, linearized Eqs. (57) and (58), we have

$$
\begin{gathered}
\widetilde{a}_{+}\left(\omega-k^{2}+2 U_{0}^{2}\right)+2 \sigma \widetilde{a}_{-} U_{0}^{2} e^{4 i U_{0}^{2}\left(t-t_{0}\right)}=0, \\
\widetilde{a}_{-}\left(-\omega-k^{2}+2 U_{0}^{2}\right)+2 \sigma \widetilde{a}_{+} U_{0}^{2} e^{4 i U_{0}^{2}\left(t-t_{0}\right)}=0,
\end{gathered}
$$

yielding

$$
\left(\omega-k^{2}+2 U_{0}^{2}\right)\left(-\omega-k^{2}+2 U_{0}^{2}\right)=4 U_{0}^{4}
$$

or, after some algebra, the linear resonance frequency $\omega=-k \sqrt{k^{2}-4 U_{0}^{2}}$ discussed previously [see Eq. (14)]. In contrast, in studying the exit from the mode conversion process, as the mode $a_{-} e^{-i \varphi}$ becomes dominant while $a_{0}$ and all other harmonics are small, the parametric driving effectively couples a different triad, $a_{0}, a_{-}, b_{-}$, while $a_{+}, b_{+}$ can now be neglected. This yields the system

$$
\begin{gathered}
i a_{0 t}+2 a_{0}\left(2 U_{0}^{2}-\left|a_{0}\right|^{2}\right)+4 a_{-}^{2} b_{-}^{*}=\frac{\mu}{2} a_{-} \\
i a_{-t}-\left(\omega+k^{2}\right) a_{-}+2 \sigma\left[a_{-}\left(2 U_{0}^{2}-\left|a_{-}\right|^{2}\right)+2 a_{0} a_{-}^{*} b_{-}\right] \\
=\frac{\mu}{2}\left(a_{0}+b_{-}\right) \\
i b_{-t}-2\left(\omega+2 k^{2}\right) b_{-}+2\left[a_{-}\left(2 U_{0}^{2}-\left|b_{-}\right|^{2}\right)+a_{-}^{2} a_{0}^{*}\right] \\
=\frac{\mu}{2} a_{-} .
\end{gathered}
$$

Here we linearize the unperturbed Eq. (62) around the final $a_{-}$ $\left(\left|a_{-}\right|=U_{0}\right)$ in the vicinity of the final time $t_{f}$ to get

$$
a_{-}=U_{0} e^{i\left(-k^{2}-\omega+4 U_{0}^{2}\right)\left(t-t_{f}\right)} .
$$

Then, seeking constant amplitude solutions $\left(a_{0}, b_{-}\right)=$ $\left(\widetilde{a}_{0}, \widetilde{b}_{-}\right) e^{i\left(-k^{2}-\omega+4 U_{0}^{2}\right)\left(t-t_{f}\right)}$ of the unperturbed, linearized Eqs. (61) and (63), we obtain

$$
\begin{aligned}
& \left(k^{2}+\omega+2 U_{0}^{2}\right) \widetilde{a}_{0}+2 U_{0}^{2} e^{2 i\left(-k^{2}-\omega+4 U_{0}^{2}\right)\left(t-t_{f}\right)} \widetilde{b}_{-}^{*}=0 \\
& \left(-3 k^{2}-\omega+2 U_{0}^{2}\right) \widetilde{b}_{-}+2 U_{0}^{2} e^{2 i\left(-k^{2}-\omega+4 U_{0}^{2}\right)\left(t-t_{f}\right)} \widetilde{a}_{0}^{*}=0
\end{aligned}
$$

yielding

$$
\left(k^{2}+\omega+2 U_{0}^{2}\right)\left(-3 k^{2}-\omega+2 U_{0}^{2}\right)=4 U_{0}^{4}
$$

or, after some algebra, the correct linear resonance frequency $\omega=-\left(2 k^{2}-k \sqrt{k^{2}-4 U_{0}^{2}}\right)$ at the end of the mode conversion problem, as discussed above within the fully nonlinear Lagrangian theory [see Eq. (38)].

Finally, we briefly discuss the defocusing $(\sigma=-1)$ case. Here we find that the autoresonant excitation is observed when one passes the linear resonant frequency $\omega=k \sqrt{k^{2}+4 U_{0}^{2}}$, while the driving frequency decreases as before. We illustrate the autoresonant dynamics from the reduced model in this case in Fig. 8 and compare with the results of full simulations. One finds that in contrast to $\sigma=1$ case, the initially flat solution is now converted into $a_{+} e^{i \varphi}$ mode, while $a_{-}$and $b_{ \pm}$(not shown in the figure) remain small throughout the conversion process and the harmonics are phase locked to the drive after passage through the linear resonance. The initial stage of this mode conversion can be again studied via the reduced set of equations describing the triad $a_{0}, a_{ \pm}$, while the final stage of the mode conversion in the $\sigma=-1$ case can be analyzed via the reduced set for the triad $a_{0}, a_{+}, b_{+}$and neglecting modes $a_{-}, b_{-}$. 

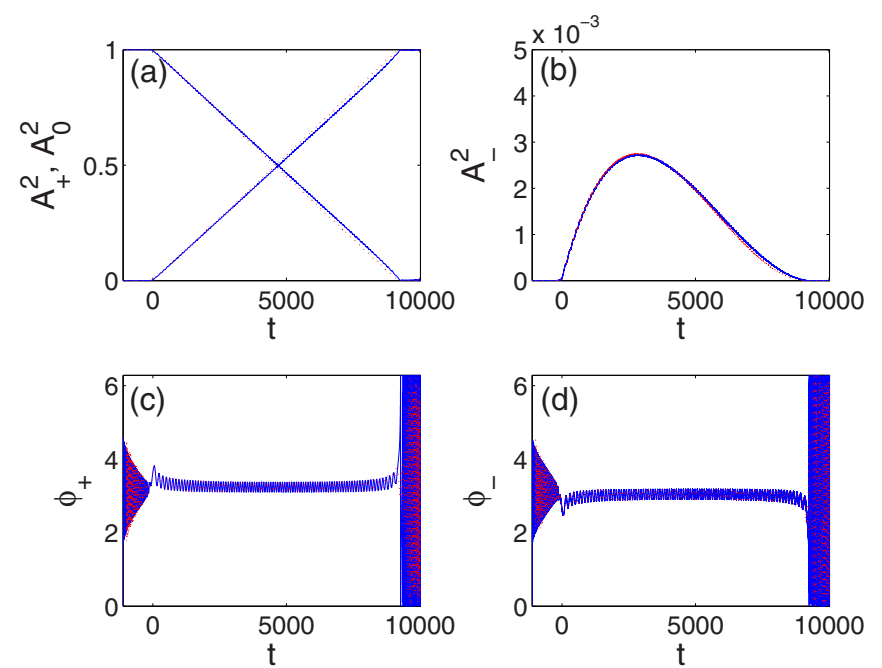

FIG. 8. The amplitudes (a b) and the relative phases (b, c) of $a_{0}, a_{+}, a_{-}$harmonics in $\sigma=-1$ example $\left(U_{0}=1, k=3, \mu=0.002\right.$, and $\alpha=-0.0005)$. The results of full simulations are shown by red dashed line.

\section{CONCLUSIONS}

In conclusion, we have studied the parametric autoresonance phenomenon in the NLS equation (1) driven by a small amplitude, chirped frequency wave. The system was analyzed for a flat ( $x$-independent) initial and periodic boundary conditions for both the focusing and defocusing signs of the nonlinearity. The analysis was based on the Whitham's averaged variational principle, allowing separation of fast and slow time scales in the problem. It was shown that the transition to autoresonance in the system takes place if the driving amplitude $\mu$ exceeds a sharp threshold scaling as $\alpha^{3 / 4}$ [see Eq. (11)], $\alpha$ being the frequency chirp rate of the driving wave. This analytic result obtained using a weakly nonlinear limit of the variational theory was in excellent agreement with numerical simulations. For studying the developed autoresonant stage of evolution, we have used the fully nonlinear version of the averaged variational principle. We have described the autoresonant quasi-equilibrium assuming the ideal phase locking in the system, as the driven wave follows the phase of the driving wave continuously, by adjusting its parameters accordingly. This theory predicted a complete conversion of the flat into a traveling wave at the end of the autoresonant evolution, the result confirmed by numerical simulations. The simulations also illustrated the stability of the quasi-equilibrium. This stability is important in applications, because it confirms the typical robustness of the autoresonant excitations regardless the form of time variation of the driving frequency as long as this variation is sufficiently slow. We have also suggested a simplified theory of wave transformations in the parametrically driven NLS system based on a truncated harmonics expansion. In particular, it was shown that the transformations in the driven system can be viewed as nonlinear interactions within triads of harmonics of the driven NLS solution. The truncated harmonics expansion theory confirmed the numerically observed stability of the parametrically autoresonant solutions. Nevertheless, the proof of stability via the fully nonlinear Whitham's variational theory is still a challenging goal for future work. Inclusion of dissipation in the problem is also important. The effect of dissipation on autoresonant excitations was studied both in theory and experiments in several dynamical problems $[28,29]$, but not in the context of nonlinear waves. It was found that weak dissipation in these problems does not affect stability of autoresonant oscillations, but changes the scaling of the autoresonant threshold with the driving frequency chirp rate. The study of autoresonant wave problems (including NLS with both the direct and the parametric driving) with dissipation seems to be an important goal for the future, which possibly can be dealt with via the dissipational version of the Whitham's variational principle. In addition, similar to the direct drive approach [23], a more general parametric driving term $V(x, t) \psi$, where $V(x, t)$ is a combination of several traveling waves with chirped frequencies, may allow autoresonant excitation and control of multiphase NLS waves [20]. Finally, the use of the parametric autoresonance in controlling solutions of other nonlinear wave systems with and without dissipation seems to be an important future direction.

\section{ACKNOWLEDGMENTS}

This work was supported by the Israel Science Foundation (Grant No. 30/14) and, partially, by 211 GRF contract 02.A03.21.0006.

\section{APPENDIX: WEAKLY NONLINEAR VARIATIONAL PROBLEM}

Our starting point is the weakly nonlinear averaged Lagrangian density derived in the direct driving problem [23] by using expansions (6) and (7):

$$
\Lambda_{0}=-\frac{\sigma}{2} M_{0}-\frac{\Omega_{0}}{4} M_{1}+\frac{\Omega}{4} M_{2}+\frac{k^{2}}{4} M_{3}+,
$$

where

$$
\begin{aligned}
M_{0}= & u_{0}^{4}+3 u_{0} u_{1}^{2} u_{2}+3 u_{0}^{2}\left(u_{1}^{2}+u_{2}^{2}\right)+3 u_{1}^{4} / 8 \\
M_{1}= & 2 u_{0}^{2}+u_{1}^{2}+u_{2}^{2} \\
M_{2}= & 2 u_{0} u_{1} v_{1}+u_{1} u_{2} v_{1}+u_{1}^{2} v_{2}+4 u_{0} u_{2} v_{2} \\
M_{3}= & u_{1}^{2}+4 u_{2}^{2}+v_{1}^{2}\left(u_{0}^{2}+3 u_{1}^{2} / 4+u_{0} u_{2}\right) \\
& +4 u_{0} u_{1} v_{1} v_{2}+u_{0}^{2} v_{2}^{2}
\end{aligned}
$$

The full weakly nonlinear averaged Lagrangian density (5) in the problem is

$$
\Lambda=\Lambda_{0}+\frac{\mu}{2} u_{0} u_{1} \cos \Phi
$$

We take the variation with respect to $u_{0}$ to get [to $O\left(u_{1}^{2}\right)$ ]

$$
2 u_{0}^{2}=-\sigma \Omega_{0}-3 u_{1}^{2} \text {. }
$$

Similarly, variations with respect to $u_{1}, u_{2}, v_{1}$, and $v_{2}$ yield four additional equations, which after elimination of $u_{2}$ and $v_{2}$ (for details of these developments see Ref. [23]) 
results in

$$
\begin{gathered}
\left(k^{2}-\Omega_{0}-6 \sigma u_{0}^{2}\right) u_{1}+\Omega u_{0} v_{1}+N_{1}=-\mu U_{0} \cos \Phi \\
\Omega u_{0} u_{1}+k^{2} u_{0}^{2} v_{1}+N_{2}=0
\end{gathered}
$$

where

$$
N_{1}=12 u_{1}^{3}\left(\frac{\rho^{2}}{32 \zeta}-\zeta\right), \quad N_{2}=\frac{3 \Omega u_{1}^{3}}{8 U_{0}}(1-8 \sigma \zeta)
$$

and $\zeta=U_{0}^{2} / k^{2}, \rho=\Omega / k^{2}$. Then we substitute $v_{1}$ from Eq. (A7) into (A6) to get

$$
\Omega^{2}-k^{2}\left(k^{2}+2 \Omega_{0}\right)+q u_{1}^{2}=\frac{\mu k^{2} U_{0}}{u_{1}} \cos \Phi,
$$

where $q=12\left(2 U_{0}^{2}-\sigma k^{2}\right)$. Next, we take variations with respect to $\Theta$ and $\xi$, yielding

$$
\left(\frac{\partial \Lambda}{\partial \Omega}\right)_{t}=\frac{1}{4} M_{2 t}=\frac{\mu U_{0}}{2} u_{1} \sin \Phi
$$

and

$$
\left(\frac{\partial \Lambda}{\partial \Omega_{0}}\right)_{t}=-\frac{1}{4} M_{1 t}=0 .
$$

From the last equation, to $O\left(u_{1}^{2}\right), M_{1}=2 u_{0}^{2}+u_{1}^{2}=U_{0}^{2}$ :

$$
u_{0}^{2}=U_{0}^{2}-u_{1}^{2} / 2 \text {. }
$$

This can be used in (A5) to get

$$
\Omega_{0}=-2 \sigma\left(U_{0}^{2}+u_{1}^{2}\right),
$$

which, after substitution into (A9), yields

$$
\Omega^{2}-\Omega_{r}^{2}+r u_{1}^{2}=\frac{\mu k^{2} U_{0}}{u_{1}} \cos \Phi .
$$

Here the linear resonance frequency $\Omega_{r}$ is defined via

$$
\begin{aligned}
& \Omega_{r}^{2}=k^{2}\left(k^{2}-4 \sigma U_{0}^{2}\right), \\
& r=-2 \sigma \Omega_{r}(3 \rho+1 / \rho),
\end{aligned}
$$

and $\rho=\Omega_{r} / k^{2}$. Next, we assume proximity to the linear resonance, $\Omega=\Omega_{r}+\delta \Omega,\left|\delta \Omega / \Omega_{r}\right| \ll 1$ and use (A14) to find

$$
\delta \Omega \approx \sigma(3 \rho+1 / \rho) u_{1}^{2}+\frac{\mu k^{2} U_{0}}{2 \Omega_{r} u_{1}} \cos \Phi .
$$

This result yields the evolution equation for the phase mismatch

$$
\Phi_{t}=\omega(t)-\Omega=\alpha t-\sigma(3 \rho+1 / \rho) u_{1}^{2}-\frac{\mu k^{2} U_{0}}{2 \Omega_{r} u_{1}} \cos \Phi .
$$

The amplitude evolution is governed by (A10),

$$
\frac{1}{4}\left(2 u_{0} u_{1} v_{1}\right)_{t} \approx \frac{\mu U_{0}}{2} u_{1} \sin \Phi,
$$

where, to lowest order $u_{0}=U_{0}$ and $v_{1}=-\left(\Omega_{r} u_{1}\right) /\left(k^{2} U_{0}\right)$ [see Eq. (A14)] and, therefore,

$$
u_{1 t} \approx-\frac{\mu k^{2} U_{0}}{2 \Omega_{r}} \sin \Phi .
$$

Equations (A18) and (A20) comprise a full set of equations in the weakly nonlinear problem.
[1] E. M. McMillan, Phys. Rev. 68, 143 (1945).

[2] V. I. Veksler, J. Phys. USSR 9, 153 (1945).

[3] L. Friedland, Scholarpedia 4, 5473 (2009).

[4] L. Friedland, Phil. Trans. R. Soc. 121, 299 (1831).

[5] A. Hasegawa, Optical Solitons in Fibers (Springer, Berlin, 1990).

[6] F. Kh. Abdullaev and S. Kh. Tadjimuratov, Opt. Commun. 116, 179 (1995).

[7] V. E. Zakharov, V. S. L'vov, and S. S. Starobinets, Sov. Phys. Usp. 17, 896 (1975).

[8] K. R. Smith, V. I. Vasyuchka, M. Wu, G. A. Melkov, and C. E. Patton, Phys. Rev. B 76, 054412 (2007).

[9] J. J. Garcia-Ripoll, V. M. Perez-Garcia, and P. Torres, Phys. Rev. Lett. 83, 1715 (1999).

[10] M. Assaf and B. Meerson, Phys. Rev. E 72, 016310 (2005).

[11] O. Ben-David, M. Assaf, J. Fineberg, and B. Meerson, Phys. Rev. Lett. 96, 154503 (2006).

[12] D. F. DuBois and M. V. Goldman, Phys. Rev. Lett. 14, 544 (1965).

[13] J. Fajans, E. Gilson, and L. Friedland, Phys. Rev. E 62, 4131 (2000).
[14] A. Kaplan, B. V. Gisin, and B. A. Malomed, J. Opt. Soc. Am. B 19, 522 (2002).

[15] M. Bondila, I. V. Barashenkov, and M. M. Bogdan, Physica D 87, 314 (1995).

[16] F. F. Chen, Introduction to Plasma Physics and Controlled Fusion (Plenum Press, New York, 1984).

[17] B. Malomed, in Encyclopedia of Nonlinear Science, edited by A. Scott (Routledge, New York, 2005), pp. 639-643.

[18] L. Pitaevskii and S. Stringari, Bose-Einstein Condensation (Clarendon, Oxford, 2003).

[19] O. Morsch and M. Oberthaler, Rev. Mod. Phys. 78, 179 (2006).

[20] Y. Ma and M. J. Ablowitz, Stud. Appl. Math. 65, 113 (1981).

[21] L. Friedland, Phys. Rev. E 58, 3865 (1998).

[22] L. Friedland and A. G. Shagalov, Phys. Rev. Lett. 81, 4357 (1998).

[23] L. Friedland and A. G. Shagalov, Phys. Rev. E 71, 036206 (2005).

[24] N. N. Bogoliubov and Y. A. Mitropolski, Asymptotic Methods in the Theory of Non-Linear Oscillations (Gordon and Breach, New York, 1961). 
[25] G. B. Whitham, Linear and Nonlinear Waves (Wiley, New York, 1974).

[26] C. Canuto, M. Y. Hussaini, A. Quarteroni, and T. A. Zang, Spectral Methods in Fluid Dynamics, Springer Series in Computational Physics (Springer, Berlin, 1988).
[27] L. Friedland, A. G. Shagalov, and S. V. Batalov, Phys. Rev. E 92, 042924 (2015).

[28] J. Fajans, E. Gilson, and L. Friedland, Phys. Plasmas 8, 423 (2001).

[29] O. Naaman, J. Aumentado, L. Friedland, J. S. Wurtele, and I. Siddiqi, Phys. Rev. Lett. 101, 117005 (2008). 